REVISION DE TEMAS

\title{
Pediculosis pubiana
}

\author{
Gerzaín Rodríguez ${ }^{1}$, Gloria Patricia Barrera ${ }^{2}$, Milton González ${ }^{3}$, Fernando Bulla ${ }^{3}$
}

\section{Definición}

La pediculosis púbica es una infestación parasitaria producida por el piojo Pthirus pubis, llamado también piojo chupador o ladilla (figura 1).

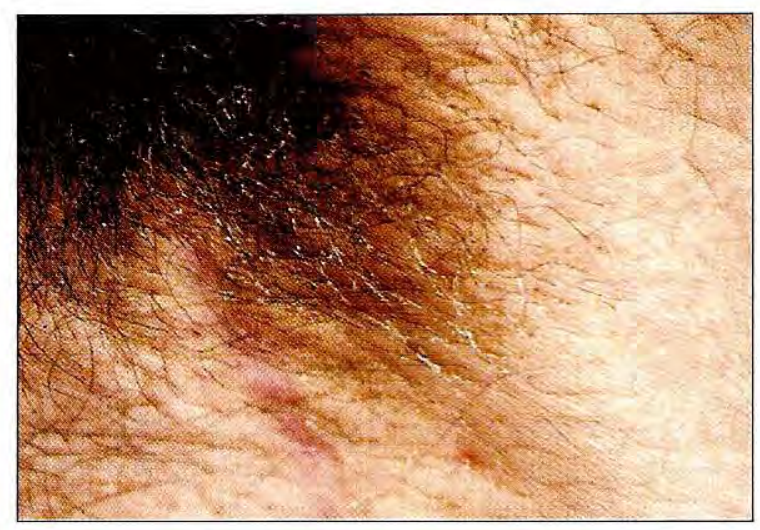

Figura 1. Pediculosis pubiana en hombre de 17 años de edad, con prurito pubiano y nudosidades en la base de los tallos pilosos.

\section{Etiología}

Los piojos son insectos chupadores, ectoparásitos de los mamíferos, pertenecientes al orden Anoplura, palabra de origen griego que significa "de cola desarmada"; carecen de alas, poseen piezas bucales para morder y sufren metamorfosis simple (1). Tienen su cuerpo dividido en cabeza, tórax y abdomen (figuras 2 y 3 ). La cabeza está provista de dientes preestomales para fijarse a la piel y de tres estiletes como aparato chupador que guardan retraidos en un saco (figura 4) (1), desde donde los introducen en los vasos de la dermis, de los cuales chupan sangre 2 a 3 veces al día $(2,3)$. Son parásitos específicos de cerdos, perros, ratas, gatos, ovejas, monos y muchos mamíferos más (4). Los piojos específicos del hombre son Pediculus humanus con dos variedades, capitis y corporis, y Pthirus pubis.

P. pubis cuando es adulto, mide 1-2 mm de longitud y es casi tan largo como ancho. Posee un par de antenas y tres pares de patas fuertes con garras muy desarrolladas en el segundo y tercer par, adaptadas para asirse firmemente a los pelos, morfología general por la cual se le ha llamado piojo cangrejo (figuras 2 y 3 ). Cuatro piececillos

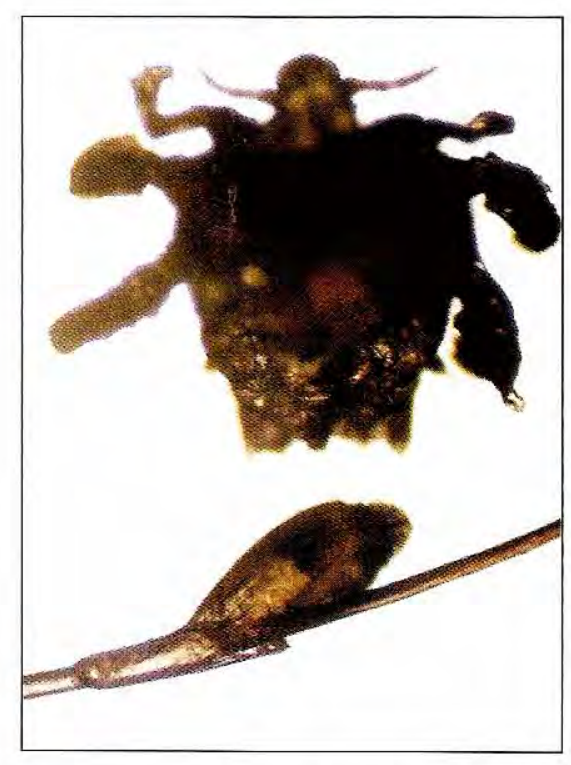

Figura 2. P. pubis adulto y liendra adherida a un tallo piloso.

\footnotetext{
1 Laboratorio de Patología, Instituto Nacional de Salud; Departamento de Patología, Facultad de Medicina, Universidad Nacional de Colombia, Santa Fe de Bogotá.

2 Laboratorio de Microscopía Electrónica, Corpoica, Santa Fe de Bogotá.

3 Residentes de Dermatología, Facultad de Medicina, Universidad Nacional de Colombia, Santa Fe de Bogotá.
} 


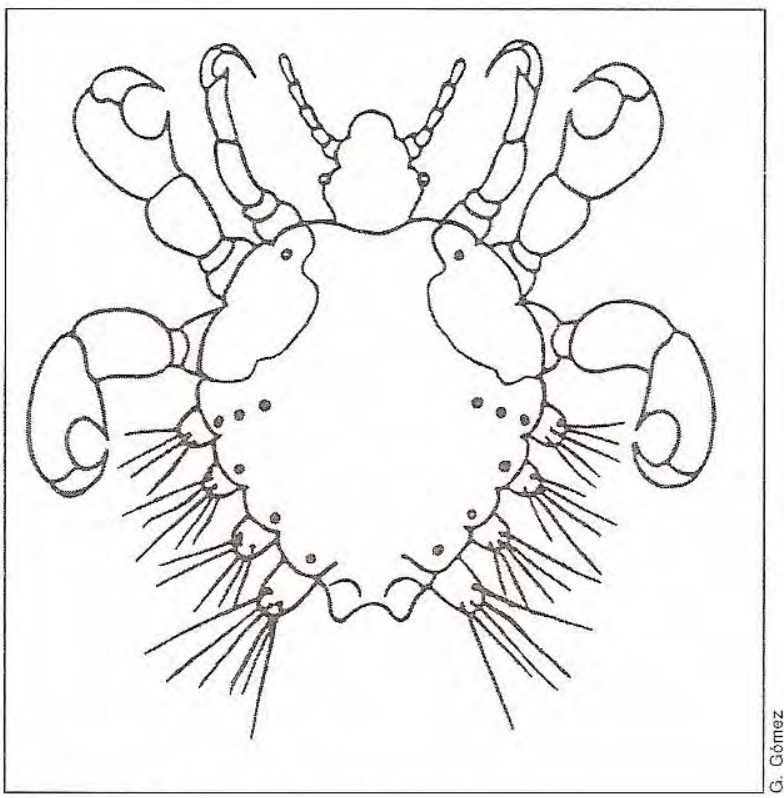

Figura 3. Esquema de $P$ pubis.

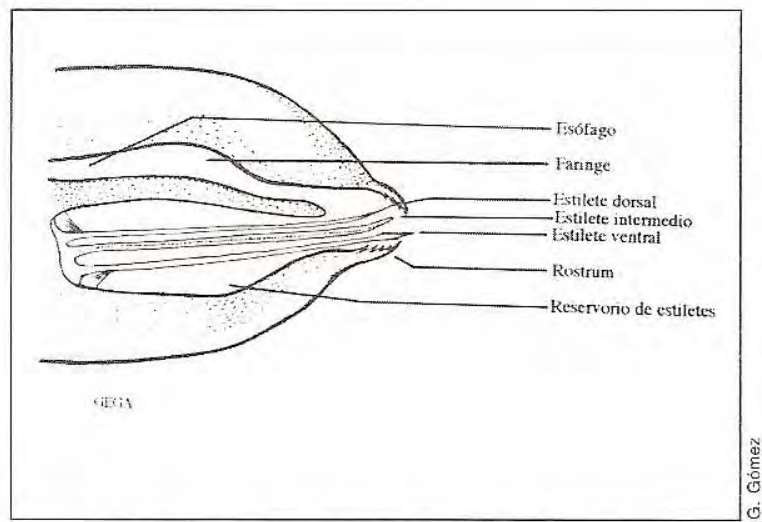

Figura 4. Aparato chupador de sangre de un piojo.

cónicos, provistos de finas cerdas, están a cada lado del cuerpo (figura 4). Su anatomía refleja una adaptación admirable a un hábitat hostil y difícil (3).

\section{Ciclo vital}

Cada hembra produce entre treinta y cincuenta huevos denominados liendres, durante su vida adulta, a razón de unos tres huevos diarios $(1,2)$. Las liendres miden 0,3-0,8 mm; son ovoides, opalescentes, con una estructura en su polo superior llamada opérculo, constituido por una serie de componentes hexagonales o aeropilos (3), con un poro central, distribuidas en dos hileras que conforman una cúpula que brinda protección y permite la oxigenación de la larva; este opérculo se desprende cuando la ninfa abandona el caparazón (figuras 5-9). Las liendres se fijan por un cemento a los pelos (figuras 2, 5 y 9 ). Luego de 7-8 días de incubación, emerge la ninfa que se adhiere al pelo y empieza a alimentarse del huésped, iniciándose así su metamorfosis incompleta para llegar a ser adulto luego de 3 mudas y de 13 a 17 días $(5,6)$.

El adulto se fija en la base de dos pelos pubianos contiguos para poder alimentarse, lo cual hace introduciendo sus estiletes en los vasos de la dermis, por lo cual es un solenófago o sea que se alimenta de los vasos (3). Es sedentario y no tiende a desplazarse (3). Por eso, su presencia en áreas distintas del vello pubiano y perineal es rara. Se ha visto en el abdomen, los mulsos, las axilas, las pestañas, las cejas, la barba y el pelo de la nuca. Esta localización se atribuye a implantación directa en estos sitios o a su transporte por toallas o por el rascado del huésped (6).

Tanto las liendres como los adultos presentan poca resistencia a las temperaturas extremas y a los climas secos, así como a la inanición, lo cual ocurre cuando se desprenden y caen en la ropa interior o en la de cama. Las ladillas son parásitos obligados, sin huéspedes intermediarios. Requieren sangre humana para sobrevivir; sin ella no resisten más de 24 horas (6).

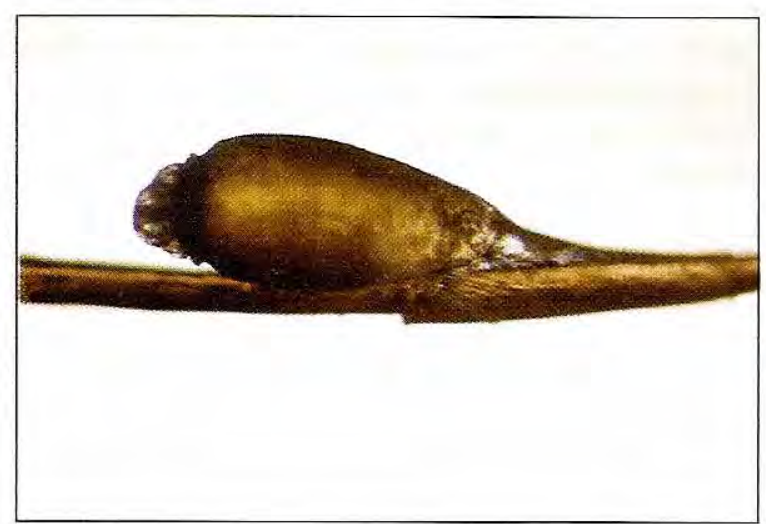

Figura 5. Liendre de $P$. pubis adherida a un tallo piloso. El opérculo, la caparazón y el material de cemento se ven con claridad. 


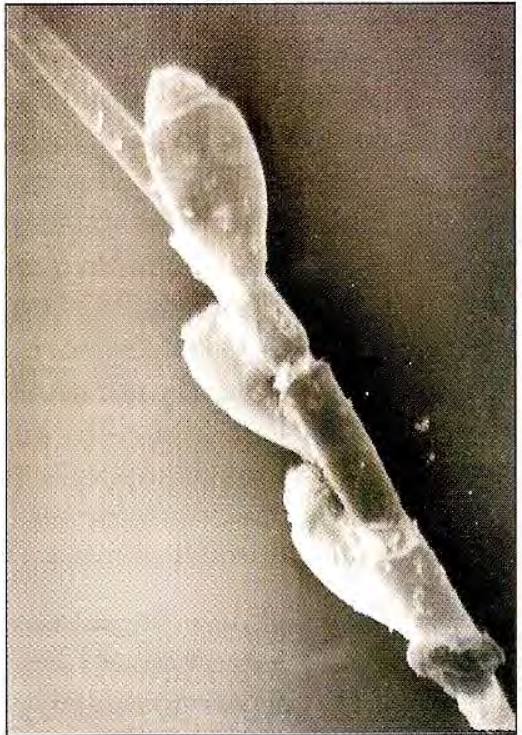

Figura 6. Microscopía electrónica de rastreo, panorámica que ilustra tres liendres de $P$. pubis adheridas a un taIlo piloso. La liendre central carece de opérculo.

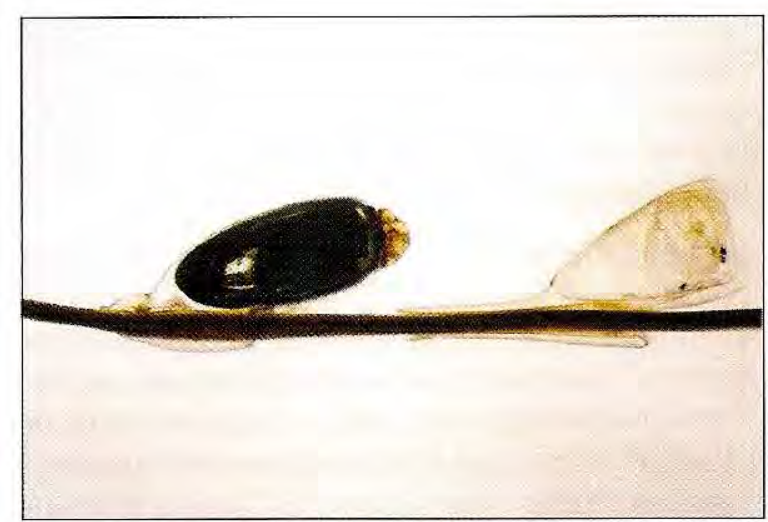

Figura 9. Tallo piloso con caparazón vacío y liendre intacta de P. pubis.

\section{Clínica}

Existe un período asintomático de, por lo menos, treinta días desde la contaminación con el parásito. En general, rara vez el paciente aloja más de 10 piojos. El síntoma principal es el prurito que es muy intenso y se debe a la picadura de los insectos. Por eso, es común encontrar lesiones secundarias por el rascado como escoriaciones,

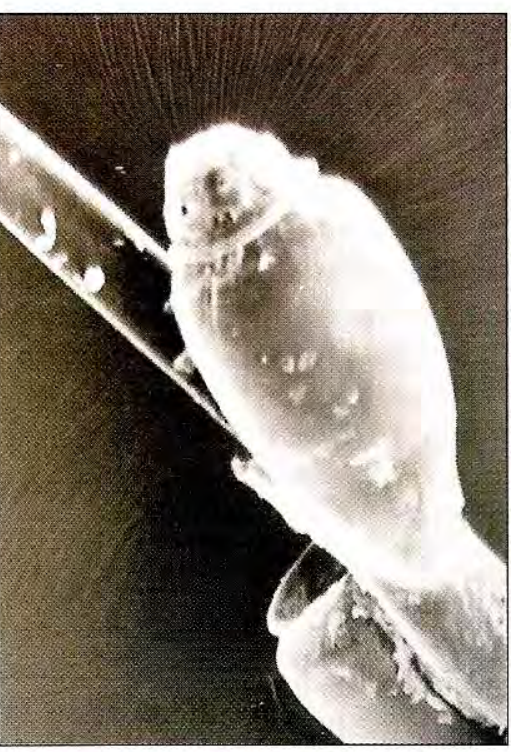

Figura 7. Microscopía electrónica de rastreo que muestra el opérculo con su corona y la adhesión al tallo piloso. La liendre inferior ha desprendido el opérculo.

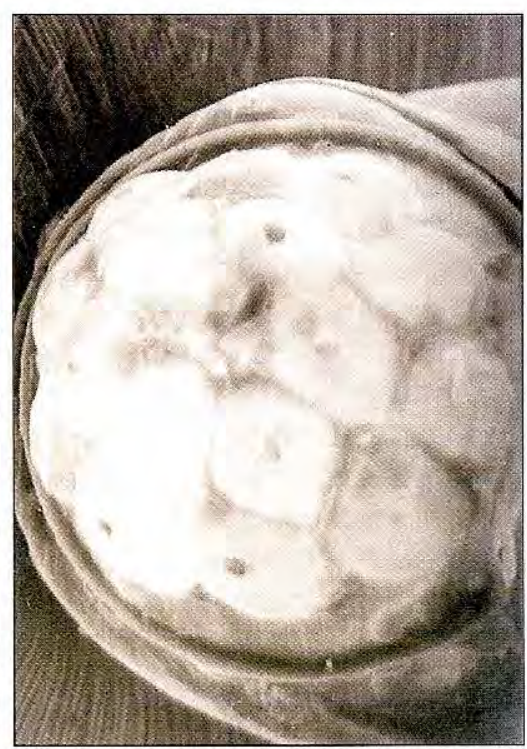

Figura 8. Aeropilos del opérculo con orificio central que permite la respiración de la larva y la inhalación de aire para romper el opérculo, por presión acumulada durante la eclosión de la ninfa (3).

liquenificación o impetiginización (figura 1) $(2,5$, 6). La piel adyacente a la picadura, especialmente cuando ocurre en los muslos, en los flancos o en el hipogastrio, puede presentar una mácula azul marrón, denominada mácula cerúlea, producida por la acción de la saliva del insecto sobre la sangre derramada en la dermis $(2,5,6)$. En la ropa interior, suelen encontrarse manchas negras, resultantes de la sangre digerida y evacuada en forma de excremento por el parásito.

El diagnóstico diferencial incluye tiñas, piedras, foliculitis, triconodosis, tricomicosis nodosa (7), eczemas, nevus melanocíticos, dermatitis cenicienta, eritrasma y sarna. El examen directo en fresco, con lupa o con microscopio, demuestra claramente las liendres y los piojos.

La blefaritis por P. pubis es ocasional y se considera un sitio usual de infestación en los niños, en los que puede ser un indicador de abuso sexual (8).

\section{Epidemiología}

Este tipo de pediculosis es casi exclusiva de los adultos y se adquiere en la mayoría de los casos 
por contacto sexual y, ocasionalmente, por fómites. Los franceses se refieren a $P$. pubis como la mariposa del amor. A diferencia de Pediculus corporis, capaz de transmitir el tifo exantemático epidémico producido por Rickettsia prowazekii, la fiebre de las trincheras, producida por Rickettsia rochalimae y la fiebre recurrente, producida por la Borrelia recurrens (4-6), P. capitis y Pthirus pubis no son transmisores de enfermedades. La prevalencia de la pediculosis pubiana se ha visto ligada a las costumbres sexuales de la sociedad (6), así como a condiciones de hacinamiento, higiene pobre y al uso compartido de sanitarios. Se notó un aumento de esta infestación con el uso de la píldora anticonceptiva hacia los años 60 (6). En Nigeria, la alta prevalencia de infestación de prostitutas con $P$. pubis ha hecho sospechar que pudiera ser vector mecánico del HIV (9). Entre nosotros, no se ha evidenciado un aumento de la infestación por estos piojos en el sida, pero no es rara su presencia, como otra de las enfermedades de transmisión sexual en estos enfermos. Como la pediculosis pubiana es una ETS, es recomendable buscar entre quienes la padezcan otras enfermedades venéreas, en primer lugar, practicando la serología para sífilis.

\section{Diagnóstico}

Los piojos y las liendres se identifican con una lupa o microscopio (figuras 2, 5 y 9). También se pueden ver mejor al colocar una linterna que ilumine lateralmente el vello pubiano. Las muestras de pelos para examen de laboratorio se pueden enviar en fresco o conservadas en etanol al $75 \%$.

\section{Tratamiento}

Históricamente se practicó el rasurado. El medicamento debe ser ovicida y ectoparaticida. En general, los huevos son más resistentes, por lo cual algunos preparados deben repetirse 7 días después de la aplicación inicial, cuando las ninfas eclosionan de las liendres. Con gran éxito se usó el DDT al 2 y $10 \%$, que fue especialmente útil para controlar epidemias de tifo epidémico (1), pero el desarrollo de resistencia al insecticida y el efecto deletéreo de éste en la naturaleza hicieron que se aboliera su uso. Otra forma de manejo es con gamabenceno o lindano, presentado como champú, crema o loción al 1\%. Luego del baño con un champú corriente, se seca el pelo bien y se aplica una onza de champú de gamabenceno, que se esparce bien y se deja actuar durante 4 minutos, después de lo cual se añade agua para hacer espuma abundante, que se enjuaga en seguida. El uso de agua y vinagre en partes iguales para enjuague ayuda a ablandar el cemento que une la liendre al pelo y su remoción se facilita con un peine fino. El gamabenceno se absorbe por la piel, pero con estas cantidades bajas y por poco tiempo se evita su toxicidad, siendo posible su uso solo una vez en embarazadas o mujeres en lactancia. No se debe usar en pacientes con historia de convulsiones ni en menores de 2 años.

Las piretrinas (Kwell ${ }^{\circledR}$-Quitoso ${ }^{\circledR}$ ) se presentan como emulsión al $0,3 \%$ o como champú al 10\%. Se usan de igual manera que el gamabenceno y han dado excelente efectividad sin efectos tóxicos (5). El malatión en loción al 0,5\% es quizás el mejor medicamento para todos los piojos (5), pero su consecución, por ser un organofosforado tóxico, no es fácil. En base acuosa, es el agente ideal para tratar las blefatiris ptiriásicas (8) que pueden representar un reto terapéutico. El petrolato por sus propiedades oclusivas ha sido útil en el control del parásito. Se afirma que el xilol (20 $\mathrm{mL}$ de xilol en $30 \mathrm{~g}$ de vaselina) es un parasiticida excelente (2).

La ivermectina es un ovicida y parasiticida eficaz, con amplio rango de acción contra varios helmintos y ectoparásitos (10). La ivermectina para uso veterinario (Ivermec ${ }^{\circledR}$ ) se ha adaptado para uso humano, disolviendo $9 \mathrm{~mL}$ del producto en $10 \mathrm{~mL}$ de champú, el cual se aplica localmente durante 3 minutos y luego se enjuaga (JG Chalela, comunicación personal). La droga para uso humano no es de disposición general en nuestro medio; se presenta en tabletas que se dan a la dosis de $100-200 \mathrm{ng} / \mathrm{kg}$. Será el medicamento de elección en la pediculosis pubiana, por analogía con lo observado en la pediculosis capitis, en la cual produjo curación de $97 \%$ de los infestados, con la dosis de $200 \mathrm{mg} / \mathrm{kg}$, por vía oral, en una sola dosis (10). Sólo tres de cien niños así tratados requirieron una segunda dosis para su curación total.

El temor fóbico o neurótico a la parasitosis puede llevar al uso excesivo de la terapia indicada, re- 
sultando en una dermatitis de contacto que llega a ser más sintomática que la pediculosis misma.

Los compañeros sexuales deben ser tratados de la misma manera. Se debe tener la precaución de usar ropa planchada y lavada con agua caliente. La liendre se destruye a $55^{\circ} \mathrm{C}$ por 10 minutos y el piojo adulto a $60^{\circ} \mathrm{C}$ por 15 minutos (2).

\section{Consejos y educación}

Cada paciente debe ser informado del diagnósti$\mathrm{co}$, del mecanismo de transmisión y de lo inocuo de la infestación, pero dando relevancia a lo que implica el riesgo de su naturaleza como enfermedad de transmisión sexual. El tratamiento y su modo de aplicación, así como su administración a la pareja sexual y las medidas higiénicas pertinentes, deben explicarse con especial claridad.

\section{Referencias}

1. Borror D, De Long D, Triplehorns C. An introduction to the study of insects. Philadelphia: Saunders College Publishing; 1981:270-4.
2. Sutton RL, Sutton RL Jr. Diseases of the skin. Tenth edition. St Louis: The CV Mosby Co.; 1939.

3. Burns DA, Sims TA. A closer look at Pthirus pubis. $\mathrm{Br}$ J Dermatol 1988;118(4):497-503.

4. Zinsser $\mathbf{H}$. Rats, lice and history. $9^{\text {th }}$ Edition. New York: Bantam Book; 1971.

5. Elgart ML. Pediculosis. Dermatologic Clin 1990;8:21928.

6. Ackerman BA. Crabs. The resurgence of Pthirus pubis. N Engl J Med 1968;278(17):950-1.

7. Scott MJ Jr, Scott MJ Sr. Nits of not? Pseudonits. Simple office diagnosis. JAMA 1980;243(22):2325-6.

8. Burns DA. The treatment of Pthirus pubis infestation of the eyelashes. Br J Dermatol 1987;117:741-3.

9. Imandeh NA. Prevalence of Pthirus pubis among sex workers in urban Jos, Nigeria. Appl Parasitol 1993; 34(4):275-7.

10. Victoria J, Aumada NS, González FG. Pediculosis capitis: tratamiento de cien niños con ivermectina. Act Terap Dermatol 1997;20:99-103. 